\title{
DESIGN OF A CHIRPED FIBER BRAGG GRATING FOR USE IN WIDEBAND DISPERSION COMPENSATION
}

\author{
İsa Navruz \\ e-mail:inavruz@eng.ankara.edu.tr
}

\author{
Ahmet Altuncu \\ e-mail: altuncu@mail.dumlupinar.edu.tr
}

Key words: Fiber Bragg grating, dispersion compensation, apodization

\begin{abstract}
A wideband chirped fiber Bragg grating (FBG) dispersion compensator operating in $\mathrm{C}$ band is designed theoretically by numerically solving the coupled mode equations. The power reflectivity spectrum and dispersion characteristics of the chirped fiber Bragg gratings are analysed. In order to achieve wideband dispersion compensation with a low insertion loss, grating length, average refractive index change, apodization profile and chirp parameter of the grating should be precisely optimized. The chirped FBG designs achieved in this study have resulted in a negative dispersion of $4.95 \mathrm{ps} / \mathrm{nm}$ for a grating length of $L=10 \mathrm{~mm}$ and a negative dispersion of 9.76 ps/nm for a grating length of $L=20 \mathrm{~mm}$ with $16 \mathrm{~nm}$ bandwidth at around $1550 \mathrm{~nm}$.
\end{abstract}

\section{INTRODUCTION}

The use of erbium doped fiber amplifiers (EDFA) in optical communication systems has made chromatic dispersion the most significant limitation for the transmission performance since EDFAs compensate for the transmission losses. The chromatic dispersion in optical fiber is a phenomenon caused by the wavelength dependence of its group refractive index. In optical fiber, the wavelength dependence of the fiber group refractive index causes a temporal broadening of the pulses as they are propagating. After a certain propagation distance, the broadening of the pulses causes a significant number of errors at the receiving end thus the information is lost. Amongst its alternatives, dispersion compensating fibers (DCF) and bulk type dispersion compensators have been widely used to compensate for the chromatic dispersion [1-2]. This scheme requires to periodically insert a few $\mathrm{km}$ of DCF with a negative dispersion coefficient in an optical link to counteract the positive dispersion effects of the conventional fiber. But the insertion of DCF increases the total loss, nonlinear effects and cost of the optical transmission system [3]. In addition, the amount of compensation is wavelength dependent and can only be perfectly achieved in a relatively narrow band. Chirped fiber Bragg grating (FBG) has also been proposed recently for chromatic dispersion compensation and is recognized as a perfect alternative do DCF. The most significant advantages of chirped FBGs are low insertion loss, nonlinear effects and cost. The idea of dispersion compensation using chirped fiber gratings was firstly proposed by Qulette [4] and later demonstrated experimentally by Williams et.al. [5]. A fiber Bragg grating is a reflective device composed of an optical fiber that contains a modulation of its core refractive index over a certain length. The grating reflects light propagating through the fiber when its wavelength corresponds to the modulation periodicity. In a chirped FBG, the periodicity of the induced index modulation varies along the grating's length. As the grating period varies along the axis, the different wavelengths are reflected by different portions of the grating and accordingly are delayed by different amounts of time. The net effect is a compression of the input pulse that can be tailored to compensate for the chromatic dispersion accumulated along the fiber link. Due to its superior advantages, the variety of fiber Bragg gratings are now widely used in optical communication systems [6] and fiber optical sensor applications [7].

\section{THEORETICAL MODEL}

The operation of fiber Bragg grating is based on the reflection of light from grating fringes and coupling of the modes. In a single mode fiber Bragg grating, the coupling occurs between the forward and backward propagating fields of the same mode. The maximum reflectivity is obtained at the wavelength providing the Bragg condition [8]:

$$
\lambda_{B}=2 n_{\text {eff }} \Lambda
$$

Here, $n_{\text {eff }}, \Lambda$ and $\lambda_{B}$, represent the effective core refractive index, grating period and Bragg wavelength, respectively. In a uniform Bragg grating having a constant grating period, the forward and backward propagating fields are given by "coupled mode equations" as [9]: 


$$
\begin{aligned}
& \frac{d U}{d z}=i \hat{\sigma} U(z)+i \kappa V(z) \\
& \frac{d V}{d z}=-i \hat{\sigma} V(z)-i \kappa^{*} U(z)
\end{aligned}
$$

Where, $U$ and $V$ are the transmitted and reflected fields respectively. In equation (2) $\kappa$ is AC coupling coefficient and $\hat{\sigma}$ is DC self-coupling coefficient and are given for single mode Bragg reflection gratings as:

$$
\begin{aligned}
& \kappa(z)=\kappa^{*}(z)=\frac{\pi}{\lambda} v \overline{\delta_{\text {neff }}}(z) \\
& \hat{\sigma}(z)=\delta+\sigma-\frac{1}{2} \frac{d \phi}{d z} \\
& \sigma=\frac{2 \pi}{\lambda} \overline{\delta n}_{\text {eff }} \\
& \delta=2 \pi n_{\text {eff }}\left(\frac{1}{\lambda}-\frac{1}{\lambda_{D}}\right)
\end{aligned}
$$

Here, $\delta$ is called as detuning parameter and is the frequency deviation ratio from the design wavelength which is taken as a reference. The design wavelength $\lambda_{D}$ is the wavelength at which the Bragg condition is satisfied when $\overline{\delta n}_{\text {eff }}$ approximates to zero and thus a maximum reflectivity occurs. The grating fringes are obtained by sinusoidal modulation of the refractive index of the fiber core with a small intensity and is described as :

$\delta n_{\text {eff }}(z)=\overline{\delta n}_{\text {eff }}(z)\left\{1+v \cos \left[\frac{2 \pi}{\Lambda} z+\phi(z)\right]\right\}$

Here, $\overline{\delta n}_{\text {eff }}$ is the average refractive index difference along the grating, $v$ is the fringe visibility of index change, $\Lambda$ is the grating period and $\phi(z)$ is the grating chirp. For a linear chirped fiber Bragg grating, the group delay and dispersion can be calculated from the phase information of power reflectivity spectrum as :

$$
\begin{aligned}
& \tau_{\rho}=\frac{d \theta}{d \omega}=-\frac{\lambda^{2}}{2 \pi c} \frac{d \theta}{d \lambda} \\
& D \rho=\frac{d \tau}{d \lambda}=\frac{2 \tau_{\rho}}{\lambda}-\frac{\lambda^{2}}{2 \pi c} \frac{d^{2} \theta}{d \lambda^{2}}
\end{aligned}
$$

Here, $\tau_{p}$ and $D_{\rho}$ represent group delay and dispersion parameters, respectively and $\theta$ is the phase of power reflectivity spectrum.

\section{NUMERICAL RESULTS}

The optical characteristics of a FBG are mainly the reflectivity spectrum, which shows the wavelength range of operation, and the group delay spectrum which provides the dispersion characteristics. The slope of the group delay spectrum is the dispersion of the device. The reflectivity spectrum of the grating can be obtained by numerically solving the coupled equation system given in (1) using matrix transfer method [10]. In Figure 1, the power reflectivity spectrum of uniform fiber reflection gratings with a $10 \mathrm{~mm}$ length is shown for different index difference $\left(\overline{\delta n}_{\text {eff }}\right)$ values.

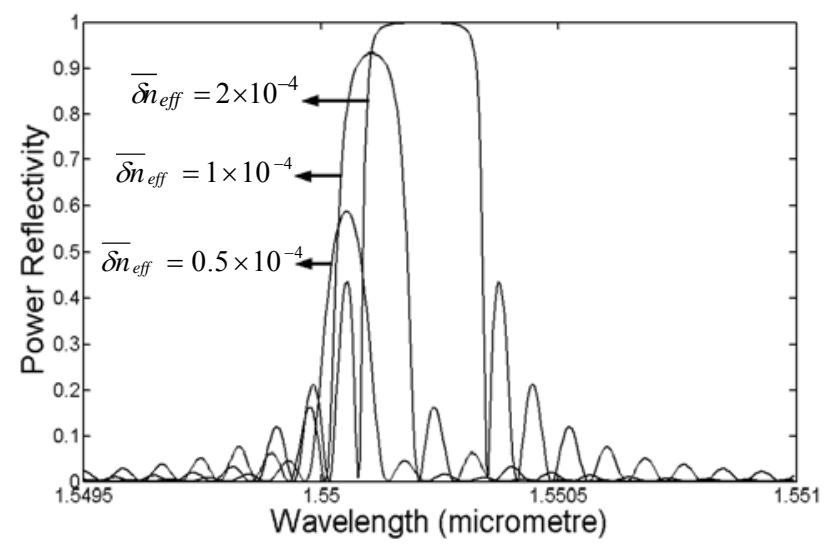

Figure 1.Power reflectivity spectrum of uniform fiber reflection gratings. $\lambda_{D}=1550 \mathrm{~nm}, L=10 \mathrm{~mm}, n_{\text {eff }}=1.447$ $\overline{\delta n}_{\text {eff }}=0.5 \cdot 10^{-4}, 1 \cdot 10^{-4}$ and $2 \cdot 10^{-4}$

As seen in this figure, the maximum reflection wavelength is shifted towards longer wavelength range when the index difference increases. The maximum wavelength deviation can be determined using

$$
\lambda_{\max }=\left(1+\frac{\overline{\delta n}_{\text {eff }}}{n_{\text {eff }}}\right) \lambda_{D}
$$

Since $\overline{\delta n}_{\text {eff }}<<n_{\text {eff }}$, the deviation from design wavelength is usually considerably small. But this deviation must be taken into account in the design of fiber grating lasers and WDM fiber reflection gratings. Standard single mode fibers operating at $1.55 \mu \mathrm{m}$ window usually have positive dispersion parameters. For a pulse propagating along an optical fiber with a positive dispersion, the short wavelength (high frequency) components of the pulse spectrum go faster than the long wavelength (low frequency) components and therefore, the pulse broadens 
with the increasing fiber length causing chromatic dispersion induced intersymbol interference (ISI) problem. It is possible to compensate chromatic dispersion using fiber Bragg gratings. In this scheme, a chirped FBG operates as a wavelength dependent dispersion compensator and light reflector. In figure 2, the power reflectivity and group delay spectrum of a linear chirped fiber reflection grating operating at around $1550 \mathrm{~nm}$ is shown.

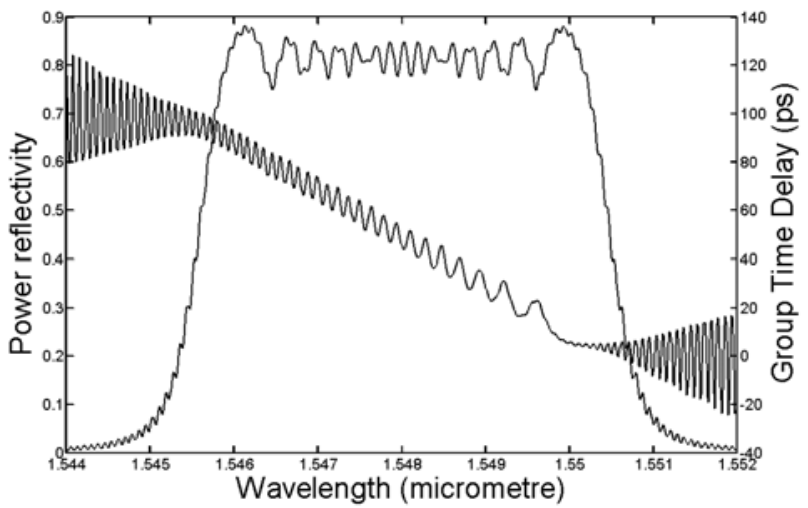

Figure 2. Power reflectivity and group delay spectrum of a linear chirped fiber Bragg grating. $\lambda_{D}=1550 \mathrm{~nm}$, $L=10 \mathrm{~mm}, \overline{\delta n}_{\text {eff }}=5 \cdot 10^{-4}, n_{\text {eff }}=1.46, \frac{d \lambda_{D}}{d z}=-2.5 \mathrm{~nm} / \mathrm{cm}$, grating period range : $530.82 \mathrm{~nm}-529.12 \mathrm{~nm}$

The power reflectivity and the group delay characteristics can be made more stable by applying apodization to fiber grating. Gaussian, Sinc and Tanh types of the apodization profile are seen in figure $3 \mathrm{a}$ and the corresponding power reflectivity spectrums are shown in figure $3 \mathrm{~b}$.

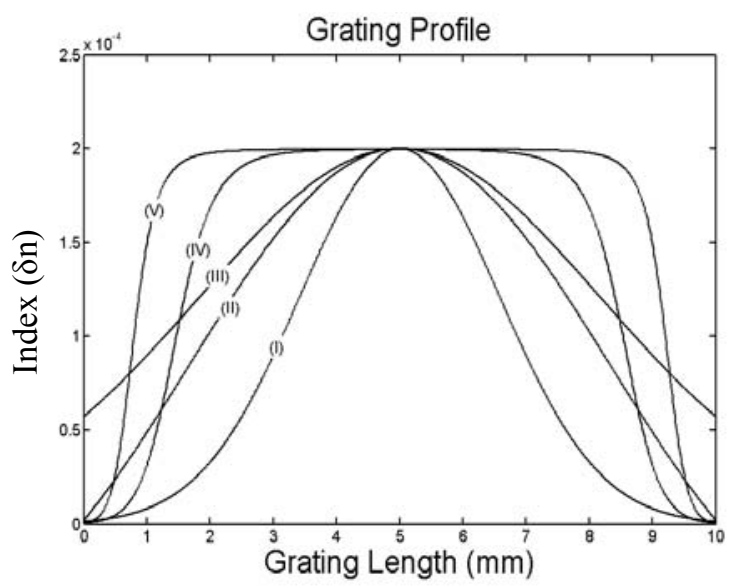

Figure 3a. Different grating apodization profiles (I) Gaussian ( $a=20)$ (II) Sinc (III) Gaussian ( $a=5)$ (IV) $\operatorname{Tanh}(\alpha=2, \beta=3)$ (V) $\operatorname{Tanh}(\alpha=4, \beta=3)$

The grating apodization profiles used are described as a function of spatial distance as [11-12-13] :
Gaussian Profile: $f(z)=\exp \left[-a\left(\frac{z-L / 2}{L}\right)^{2}\right]$

Sinc Profile: $\quad f(z)=\sin c\left(\frac{z-L / 2}{F W H M}\right)$

Tanh Profile:

$$
f(z)=\frac{1}{2}\left[1+\tanh \left(\beta\left(1-2\left|\frac{2 z}{L}\right|^{\alpha}\right)\right]\right.
$$

Where, $a$ is the Gauss width parameter, FWHM is the full width at half maximum profile width, $\alpha$ and $\beta$ are the tanh width parameters and $\mathrm{L}$ is the grating length.

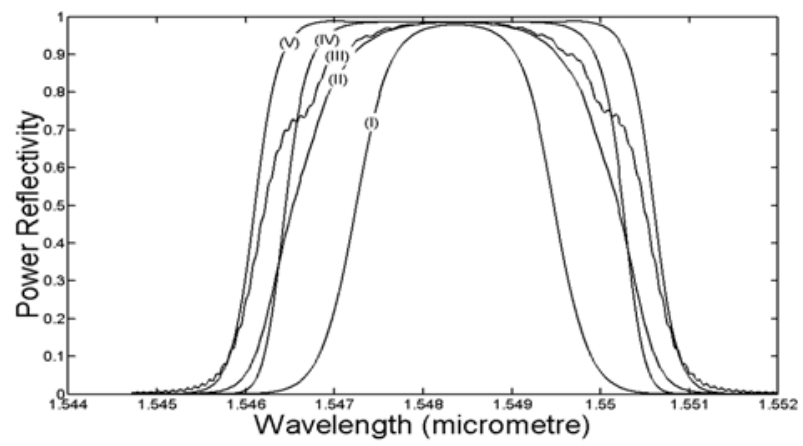

Figure 3b. Power reflectivity spectrum for different grating apodization profiles. $\lambda_{D}=1550 \mathrm{~nm}, L=10 \mathrm{~mm}$, $\overline{\delta n}_{\text {eff }}=8 \cdot 10^{-4}, n_{\text {eff }}=1.46, \frac{d \lambda_{D}}{d z}=-2.5 \mathrm{~nm} / \mathrm{cm}, v=100$

Figure 3.b shows the power reflectivity spectrum of a chirped FBG for different grating profiles. It can be seen that when the fiber grating is apodized with $\tanh (\alpha=4$, $\beta=3$ ) profile $(\mathrm{V})$, the reflection band is more stable and wider in contrast to the other profiles. The power reflectivity $(\mathrm{dB})$ and group delay spectrum of a FBG apodized with this profile is shown in figure 4.

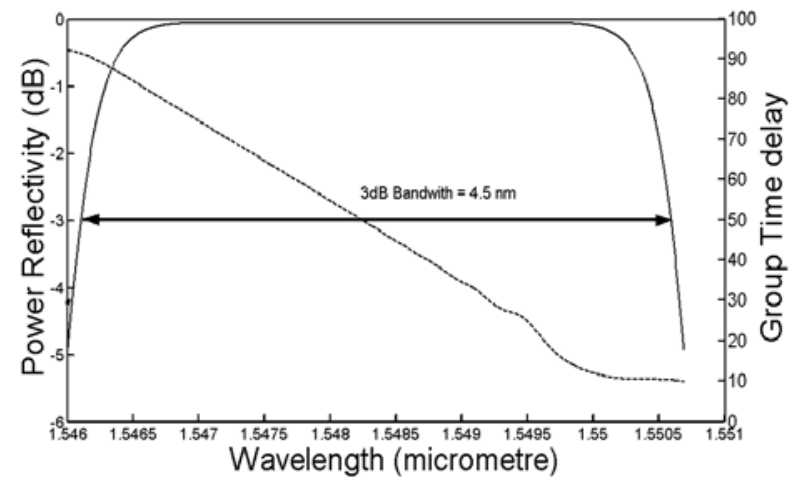

Figure 4. Power reflectivity (solid line) and group delay (dashed line) spectra of a chirped FBG apodized with Tanh $(\alpha=4, \beta=3)$ grating profile. 
The Reflection band of a chirped FBG can be more broadened by increasing chirp parameter which is given by $\frac{d \lambda_{D}}{d z}$. The grating reflection spectra for two different chirp parameters are shown in figure 5. For a chirp parameter of $-6 \mathrm{~nm} / \mathrm{cm}$, the grating period decreases from $535.59 \mathrm{~nm}$ to $531.48 \mathrm{~nm}$ linearly whereas for a chirp parameter of $-10 \mathrm{~nm} / \mathrm{cm}$, this variation occurs between $535.59 \mathrm{~nm}$ and $528.77 \mathrm{~nm}$. The reflection bandwidth increases with increasing chirp parameter but in this case the reflection power decreases.

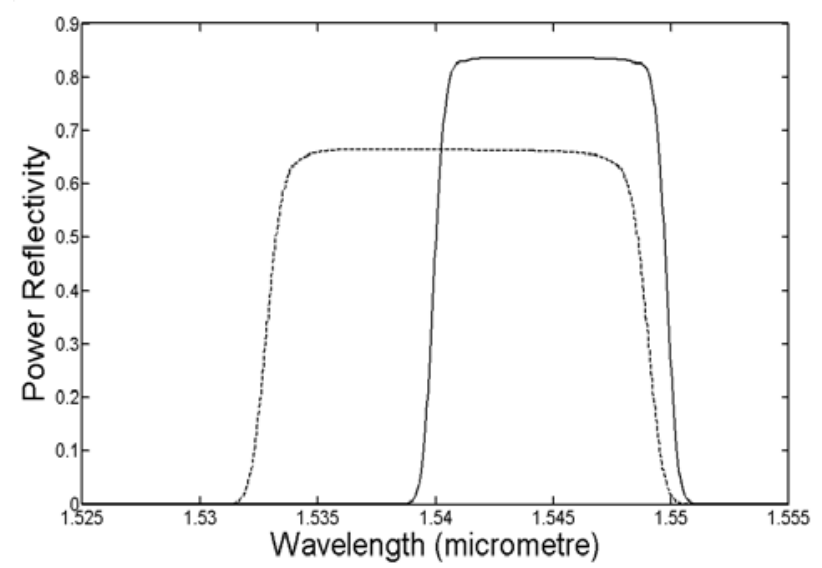

Figure 5. Power reflectivity spectrum of grating for two different chirp parameters: $-6 \mathrm{~nm} / \mathrm{cm}$ (solid line), -10 $\mathrm{nm} / \mathrm{cm} \quad$ (dashed line). $\mathrm{L}=10 \mathrm{~mm}, \quad \overline{\delta n}_{\text {eff }}=8 \cdot 10^{-4}$ $\lambda_{D}=1550 \mathrm{~nm}, \quad n_{\text {eff }}=1.447, \mathrm{v}=100$, tanh apodization $(\alpha=4, \beta=3)$.

In order to compensate for this extra power loss, the grating length should be increased for the reduced chirp parameters. The power reflectivity spectra of two different gratings with the chirp parameters of $-10 \mathrm{~nm} / \mathrm{cm}$ and $-5 \mathrm{~nm} / \mathrm{cm}$ are shown in figure 6 . As seen in this figure, if the grating length is increased to twice and the chirp parameter is reduced to half of its previous values, the reflection spectrum stays nearly same but the power reflectivity increases. The power reflectivity and group delay spectra of two different grating designs are shown in figure 7 .

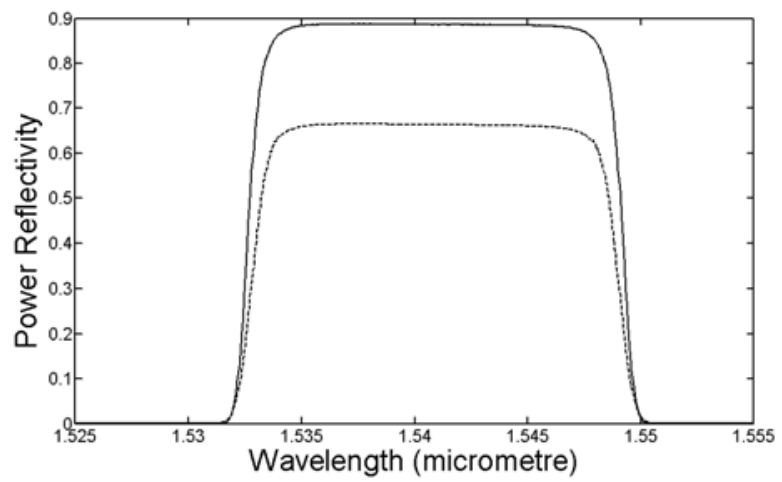

Figure 6. Power reflectivity spectra of two different grating designs, a) Chirp parameter $=-5 \mathrm{~nm} / \mathrm{cm}$ and $\mathrm{L}=$ $20 \mathrm{~mm}$ (solid line), b) Chirp parameter $=-10 \mathrm{~nm} / \mathrm{cm}$ and $\mathrm{L}=10 \mathrm{~mm}$ (dashed line).

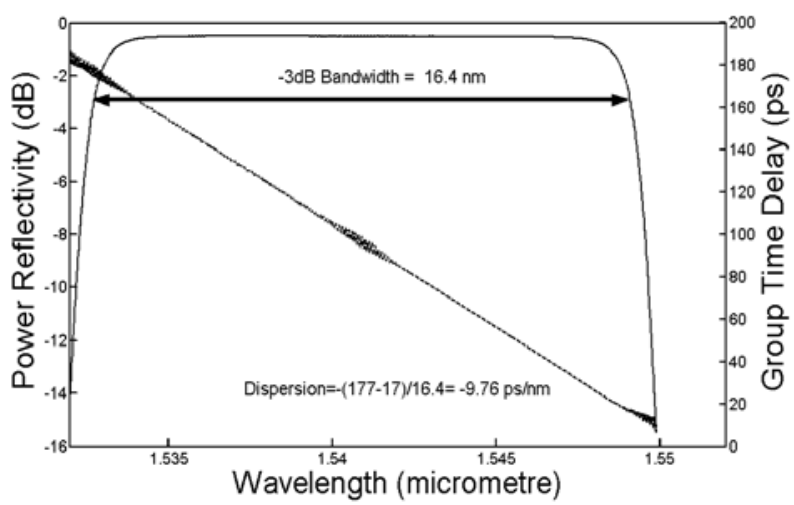

(a)

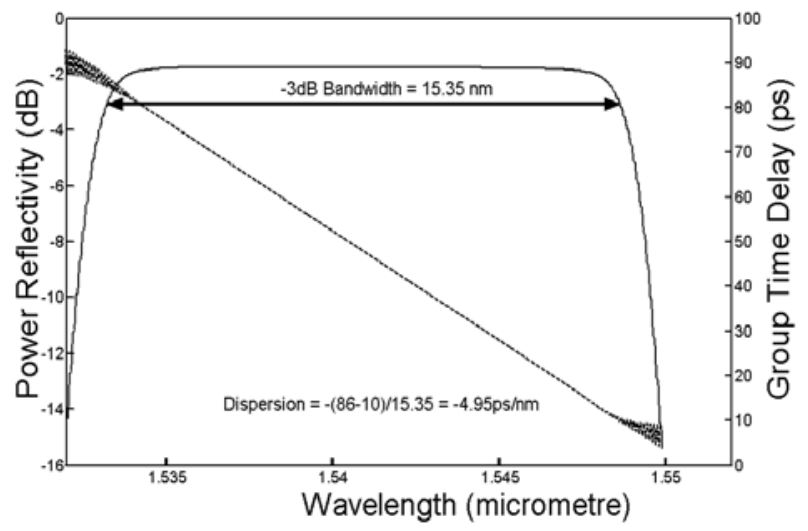

(b)

Figure 7. Power reflectivity (solid line) and group delay (dashes line) spectra of two different grating designs: a) Chirp parameter $=-5 \mathrm{~nm} / \mathrm{cm}$ and $\mathrm{L}=20 \mathrm{~mm}$ (solid line), b) Chirp parameter $=-10 \mathrm{~nm} / \mathrm{cm}$ and $\mathrm{L}=10 \mathrm{~mm}$ (dashed line). 


\section{RESULTS AND DISCUSSION}

A wideband chirped fiber Bragg grating dispersion compensator operating in $\mathrm{C}$ band was designed theoretically by numerically solving the coupled mode equations. The effects of grating length and chirp parameter of the FBG on power reflectivity and group delay spectra are investigated in detail. The results obtained have shown that increasing the chirp parameter broadens the reflection spectrum but decreases the reflectivity power for a certain grating length. On the other hand, if the grating length increases, group delay of the reflection band and therefore dispersion compensated bandwidth increases. The chirped FBG designs achieved in this study have resulted in a negative dispersion of $4.95 \mathrm{ps} / \mathrm{nm}$ for a grating length of $\mathrm{L}=10 \mathrm{~mm}$ and $\mathrm{a}$ negative dispersion of $9.76 \mathrm{ps} / \mathrm{nm}$ for a grating length of $\mathrm{L}=20 \mathrm{~mm}$ with a bandwidth of about $16 \mathrm{~nm}$ at around $1550 \mathrm{~nm}$. According to these results, a higher group delay and a wider bandwidth covering all of the $\mathrm{C}$ or $\mathrm{L}$ bands in optical fiber can be achieved by precisely optimizing the grating length, the average refractive index deviation, the apodization profile and the grating chirp parameter.

\section{REFERENCES}

1. A. M. Vengsarkar and W. A. Reed, "Dispersioncompensating single-mode fibers: efficient designs for first and second-order compensation," Opt. Lett. 18, 924926, (1993).

2. L. G. Nielsen, S. N. Knudsen, "Dispersion Compensating Fibers", Optical Fiber Technology 6, 164180, 2000.

3. G.P. Agrawal, Optical Fiber Communications Systems, John Wiley, 1997.

4. F. Qullette, "Dispersion cancellation using linearly chirped Bragg grating filters in optical waveguides" Opt. Lett. 12, 847-849, 1987.

5. J. A. R. Williams, I. Bennion, K. Sugden, and N. J. Doran, "Fiber dispersion compensation using a chirped in fiber Bragg grating," Electron. Lett. 30, 985-987, 1994.

6. F. Quellette, "Fiber Bragg Gratings help WDM push limits of fiber capacity", Laser Focus World, pp. 83-89, March 1998.

7. A. D. Kersay, M. A. Davis, H. K. Patrick, M. LeBlanc, K. P. Koo, C. G. Askins, M. A. Putnam, "Fiber grating sensors", Journal of Lightwave Technology, vol. 15, pp. 1442-1463, 1997.

8. K. O. Hill, D.C. Johnson, F. Bilodeau, "Narrow bandwidth optical waveguide transmission filters: A new design concept and applications to optical fibre communication”, Electronics Letters, vol. 23, 1987.

9. T. Erdogan, "Fibre grating spectra", Journal of Lightwave Technology, vol.15, no.8, 1277-1294, 1997.

10. A. Othonos and K. Kalli, "Fiber Bragg Gratings: Fundamentals and Applications in Telecommunications and Sensing", Artech House, Inc., 1999.
11. K. Ennser, M. N. Zervas, R. I. Laming, "Optimization of Apodized Linearly Chirped Fiber Gratings for Optical Communications", IEEE Journal Of Quantum Electronics, vol. 34, no. 5, 770-778, 1998.

12. D. Pastor, J. Capmany, D. Ortega, V. Tatay, J. Marti, "Design of Apodized Linearly Chirped Fiber Gratings for Dispersion Compensation", Journal Of Lightwave Technology, vol. 14, no. 11, 2581-2587, 1996. 13. 2. Y. Sun, C. Yun, J. Lin, Y. Qian, B. Bai, Y. Yang and W. Qiu, "Study on the apodized function of chirped fibre grating for dispersion compensation", Journal of Optoelectronics Laser, vol.10, no.3, 228-231,1999. 